\title{
Erratum to: Evaluating perceptual integration: uniting response-time and accuracy-based methodologies
}

\author{
Ami Eidels • James T. Townsend • Howard C. Hughes • \\ Lacey A. Perry
}

Published online: 3 December 2014

(C) The Psychonomic Society, Inc. 2014

Erratum to: Atten Percept Psychophys

DOI 10.3758/s13414-014-0788-y

Some of the tables and figures in the original article were inadvertently misplaced. A corrected version of the article was uploaded with figures and tables in the appropriate locations.

The online version of the original article can be found at http://dx.doi.org/ 10.3758/s13414-014-0788-y.

A. Eidels $(\triangle)$

School of Psychology, University of Newcastle, Callaghan, New

South Wales 2308, Australia

e-mail: ami.eidels@newcastle.edu.au

J. T. Townsend $\cdot$ L. A. Perry

Indiana University, Bloomington, IN, USA

H. C. Hughes

Dartmouth College, Hanover, NH, USA 